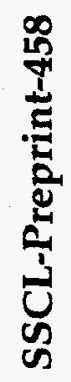

SSCL-Preprint-458

June 1993

Distribution Category: 414

H. Fenker

T. Regan

J. Thomas

Higher Efficiency Active Quenching Circuit for Avalanche Photodiodes

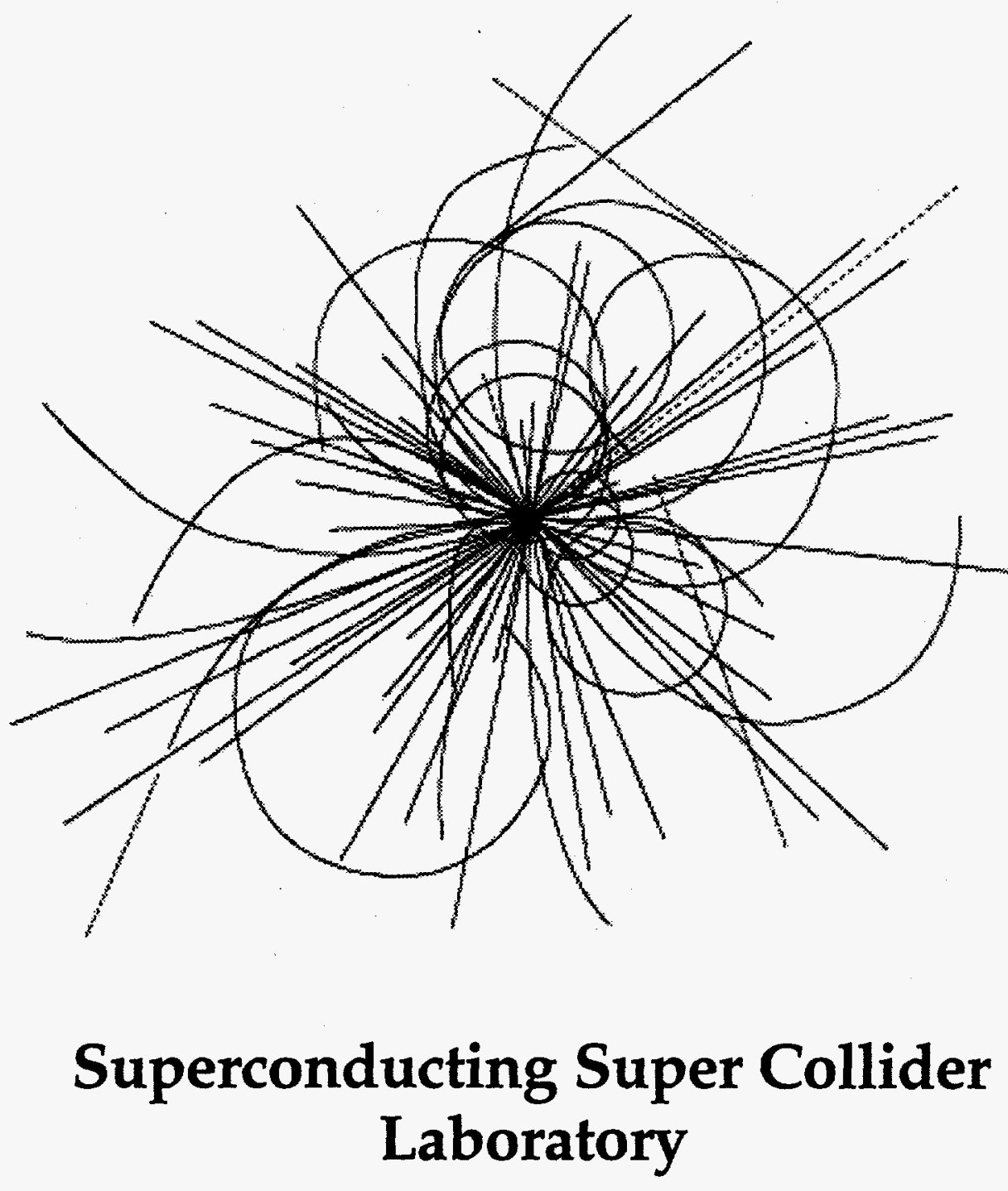

APPROVED FOR RELEASE C?

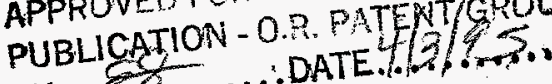
BY... SA....... DATE... 


\section{Disclaimer Notice}

This report was prepared as an account of work sponsored by an agency of the United States Govemment. Neither the United States Government of any agency thereot, nor any of their employees, makes any warranty, express or implied, or assumes any legal liability or responsibility for the accuracy, completeness, or usefulness of any information, apparatus, product, or process disclosed, or represents that its use would not infringe privately owned rights. Reterence herein to any specific commercial product, process, or service by trade name, trademark, manufacturer, or otherwise, does not necessarily constitute or imply its endorsement, recommendation, or favoring by the United States Government or any agency thereot. The views and opinions of authors expressed herein do not necessarily state or reflect those of the United States Govemment or any agency thereof

Superconducting Super Collider Laboratory is an equal opportunity employer. 


\section{DISCLAIMER}

Portions of this document may be illegible in electronic image products. Images are produced from the best available original document. 


\title{
Higher Efficiency Active Quenching Circuit for Avalanche Photodiodes*
}

\author{
H. Fenker, T. Regan, J. Thomas, and M. Wright \\ Superconducting Super Collider Laboratory ${ }^{\dagger}$ \\ 2550 Beckleymeade Ave. \\ Dallas, TX 75237
}

June 1993

\footnotetext{
*To appear in the ICFA Instrumentation Bulletin.

${ }^{\ddagger}$ Operated by the Universities Research Association, Inc., for the U.S. Department of Energy under Contract No. DE-AC35-89ER40486.
} 
Higher Efficiency Active Quenching Circuit for Avalanche Photodiodes

\author{
H.Fenker, T. Regan, J. Thomas, and M. Wright \\ Superconducting Super Collider Laboratory ${ }^{\dagger}$
}

An improved circuit for actively quenching and recharging avalanche photodiodes (APDs) which allows them to be biased to at least $30 \mathrm{~V}$ above breakdown is presented. Using this circuit it is possible to achieve the high single photon detection efficiency inherent in Geiger mode APDs while maintaining a modest deadtime. The circuit is described and observed characteristics of the system are shown.

\section{Introduction.}

It has been reported previously ${ }^{1}$ that avalanche photodiodes (APDs) may be operated at high rates (less than $50 \mathrm{~ns}$ between signals) in the Geiger mode (bias voltage higher than breakdown voltage) to produce large signals from single photoelectrons. A significant limitation of that circuit has been that the bias voltage, $V_{\text {bias }}$, could not exceed approximately $V_{\text {breakdown }}+5 \mathrm{~V}$. This limitation restricted the photoelectron breakdown probability to less than about $20 \%$ as predicted by Lightstone and McIntyre ${ }^{2}$. While still useful for detecting relatively intense bursts of light (more than about 25 photons), the system described earlier was not useful for such applications as scintillating fiber tracking, for example.

Below we report on enhancements to the previous circuit which provide proper conditions for the APD to become an efficient detector of light flashes composed of only a few (five or more) photons. The type of APD used is the CA30902S produced by EG\&G Optoelectronics. ${ }^{3}$

\section{Fast Quench and/or Recharge Circuits for Geiger Operation}

The original circuit described in reference 1 serves as a model of active quench/recharge circuit operation. It is shown in Figure 1. In the absence of a signal, the APD is biased above breakdown and is current limited by a large series resistance. Both the input to the comparator and the output of the 74HC365 tri-state buffer present high impedances at this time. When the beginning of an APD breakdown pulse is detected by the comparator, the tri-state buffer is enabled to provide a low impedance output. It switches the low-voltage side of the APD up by a TTL "high" logic level, thus reducing the voltage across the APD by the same amount. After a delay time determined by R1C1 the TTL output goes "low", restoring the full bias voltage across the APD. Finally, the buffer is disabled and the APD sees only high impedances.

The modified circuit shown in Figure 2 works in essentially the same way, but provides a higher voltage quenching pulse. It is not sufficient to simply replace the TTL tri-state

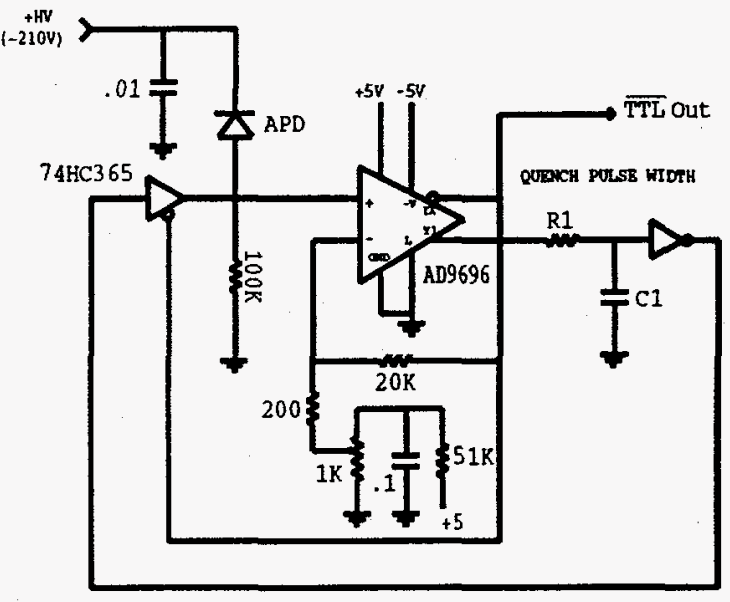

Figure 1. Original Active Quench/Recharge Circuit

buffer of Figure 1 with a higher voltage device as this high voltage pulse would adversely affect the input circuitry of almost any sensitive comparator, driving it outside the allowed operating range. The procedure we have adopted produces a higher voltage pulse but prevents the full pulse from reaching the comparator. When the comparator fires, but before the quench pulse has been developed, the comparator input is shunted to ground through the "on" resistance of a DMOS FET ( $-50 \Omega)$. In combination with a $2 \mathrm{~K}$ series resistance, this limits the voltage seen by the comparator to about $2 \%$ of the quench voltage. The quench pulse is itself formed by sequential operation of FET switches controlled by timing units and initiated by detection of a signal by the comparator. The quench potential is set by the quench supply voltage. The circuit of Figure 2 is a demonstration circuit; it would be possible to reduce the component count with further effort.

\section{Test Results}

To test the circuit we exposed the avalanche photodiode to flashes of light consisting of small numbers of photons. Early tests demonstrated that the system was ready to respond to a second flash less than $100 \mathrm{~ns}$ after the first one. While no real effort has been made to improve upon this response time, it is already fast enough to be interesting for many currently envisioned applications in high energy

Operated by Universitites Research Association Inc., for the U.S. Department of Energy under Contract No.

DE-AC35-89ER40486 


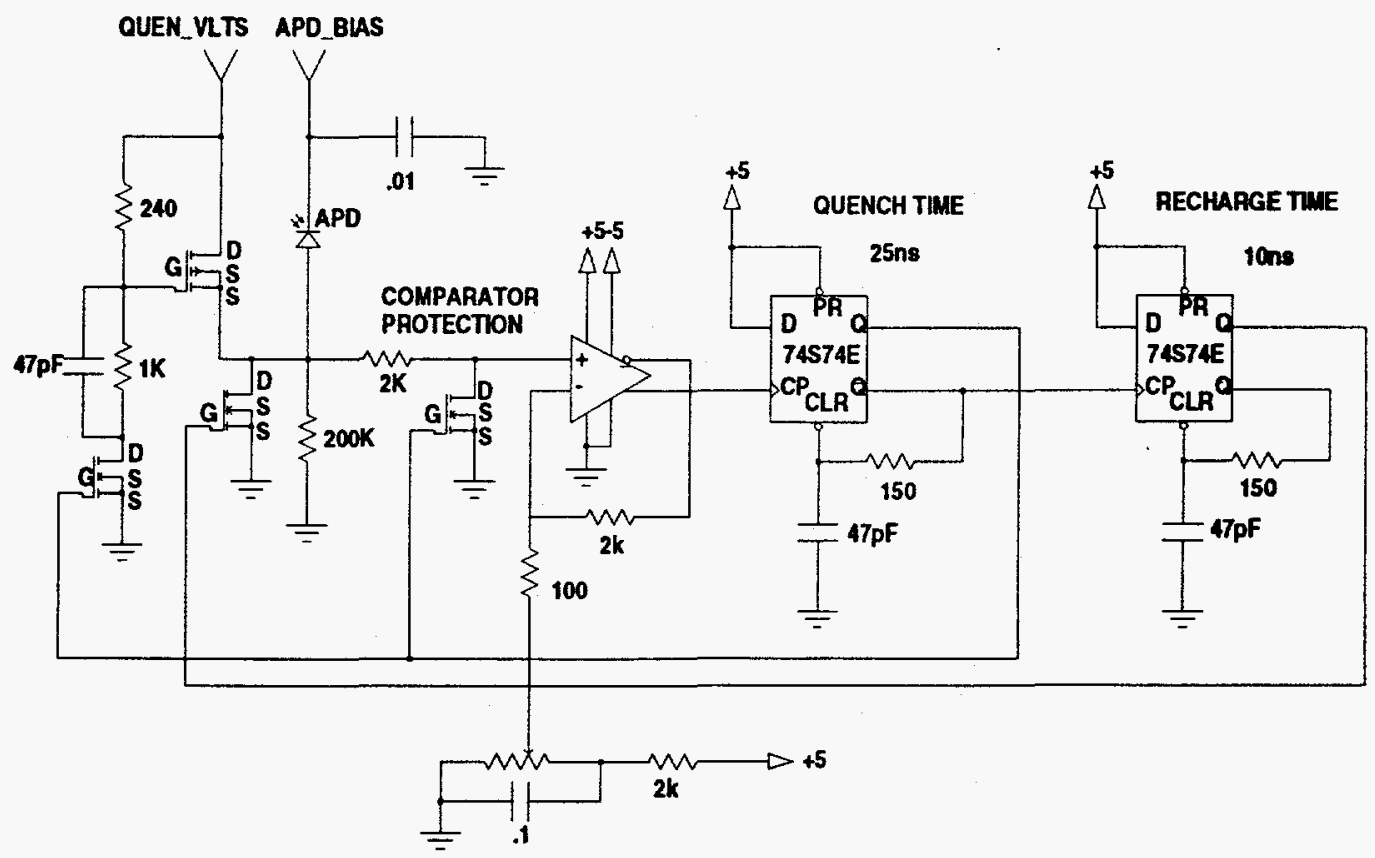

Figure 2. High Voltage Quench/Recharge Circuit

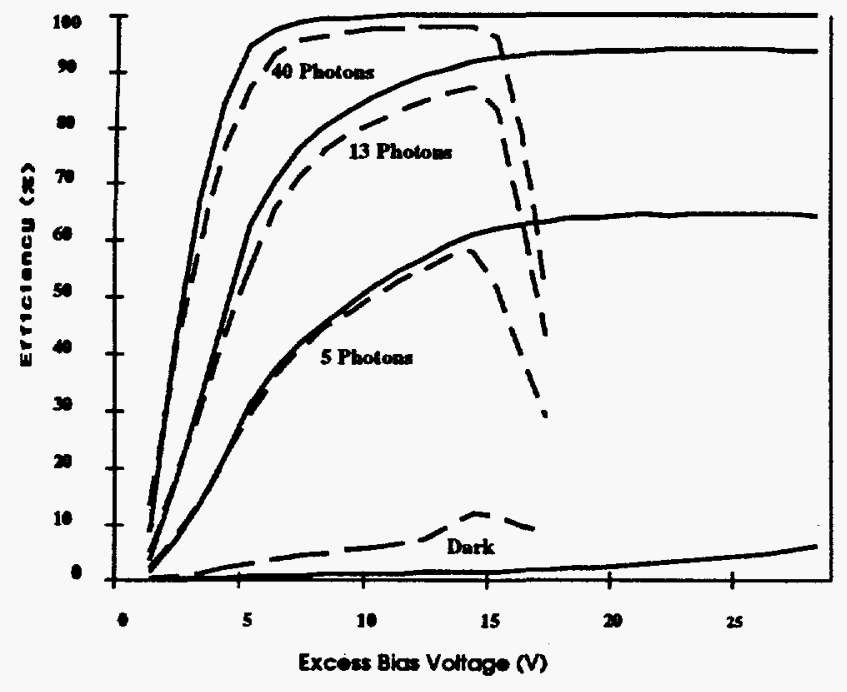

Figure 3. Efficiency Behavior of Geiger APD in HighVoltage Quench/Recharge Circuit as a Function of Voltage above Breakdown. Dashed lines show behavior in a simple passive circuit. Actual numbers of photons striking the APD are less than $40 \%$ of those shown due to an optical inefficiency at the face of the APD..

physics. Further, the demonstrated speed is not near any fundamental limit and could certainly be improved upon.

Figure 3 shows the efficiency of the APD and quench / recharge circuit for detecting light flashes. Both the intensity of the light flashes and the bias voltage of the avalanche photodiode were varied as shown. A high efficiency plateau is achieved using the active circuit at a bias voltage of about $\mathrm{V}_{\text {breakdown }}+12$ volts. For comparison we show results from similar tests performed with the APD in a passive circuit. Since the APD remains in a breakdown state for as much as several microseconds if not actively quenched, the flash detection efficiency is lower because there is a larger chance that the APD is already conducting when the test flash occurs. This effect becomes quite apparent at about $15 \mathrm{~V}$ above breakdown where the passive circuit efficiency plummets and the active efficiency remains high.

As indicated in the caption of Figure 3, the actual numbers of photons striking the active area of the APD is at most $40 \%$ of those shown because of optical inefficiencies in our experimental setup. If we take the efficiency at plateau as a measure of the mean number of photons detected at each flash intensity setting (assuming the numbers of photons per pulse obey a Poisson distribution), then the overall efficiency of the system (optical - detection) is about $18 \%$. This is in good agreement with our measured peak optical efficiency and a 50\% Detected Quantum Efficiency for the silicon APD.

\section{Calibrated Light Source}

In order to probe the performance of the system of APD and controlling circuitry as described above, one needs a source of light pulses of known intensity. We used a pulsed infrared laser diode as the light source, and determined its intensity with a commercially calibrated PIN photodiode. An interesting number of photons/flash for testing the APD is from one to about twenty. To "count" this few photons we produced many pulses per 


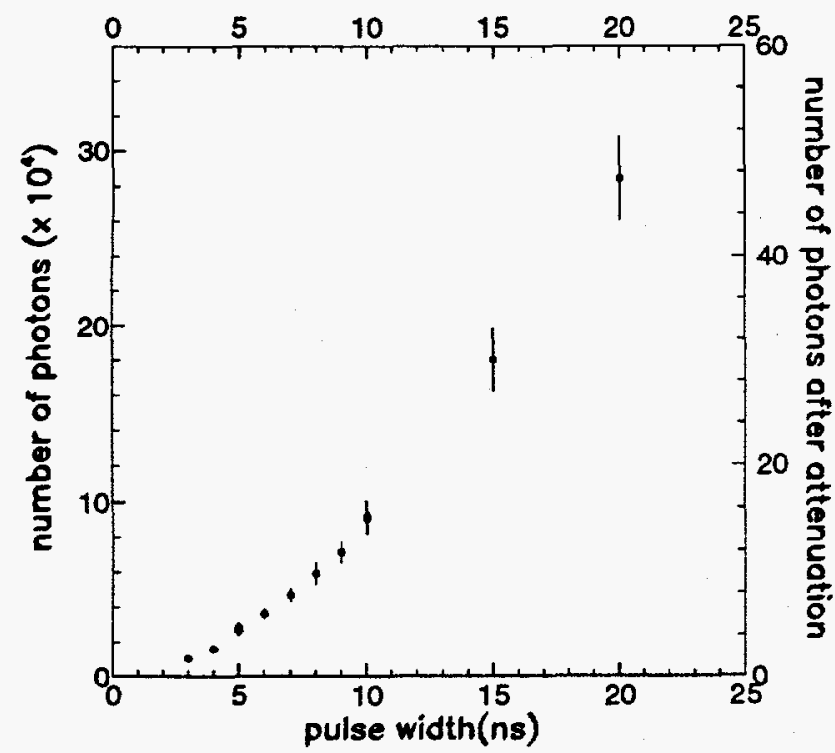

Figure 4. Number of Photons per Pulse as a function of pulse width.

second and determined the time-average photon flux incident on the PIN diode. We were able to measure pulses with intensity as low as a few thousand photons per pulse in this way. By passing the light through a known attenuator (a small integrating sphere) the flash was reduced to a few photons per pulse by the time it reached the APD. Although adjustment of the laser diode output intensity could have been used for fine adjustment of the flash brightness, we chose to adjust the time duration of the flash for convenience. Figure 4 shows the number of photons per pulse emitted from our $1 \mathrm{~mm}$ diameter plastic fiber as a function of laser diode control pulse width.

\section{Summary}

A circuit which actively quenches and recharges avalanche photodiodes with as much as $30 \mathrm{~V}$ has been developed and tested. The circuit allows efficient detection of light flashes separated by as little as $100 \mathrm{~ns}$ and composed of as few as five photons.

\section{References}

[1] T. Regan, et al., Nucl. Instr. and Meth. in Physics Research A326 (1993) 570-573.

[2] A. W. Lightstone and R. J. McIntyre, Proceedings of the OSA Topical Conference on Photon Correlation Techniques and Applications (1988).

[3] EG\&G Optoelectronics Division, 22001 Dumberry, Vaudreuil, Quebec, Canada J7V 8P7. 